\title{
Should lung biopsies be performed in patients with severe asthma?
}

\author{
Daniel Doberer ${ }^{1,2}$, Humberto E. Trejo Bittar ${ }^{3}$ and Sally E. Wenzel ${ }^{1}$ \\ Number 4 in the series "Asthma" \\ Edited by Pascal Chanez and Marc Humbert
}

\begin{abstract}
Affiliations: ${ }^{1}$ University of Pittsburgh Asthma Institute at UPMC, Pittsburgh, PA, USA. ${ }^{2}$ Dept of Internal and Pulmonary Medicine, Wilhelminenspital Wien, Medical University of Vienna, Vienna, Austria. ${ }^{3}$ Dept of Pathology, University of Pittsburgh, Pittsburgh, PA, USA.
\end{abstract}

Correspondence: Daniel Doberer, Dept of Internal and Pulmonary Medicine, Wilhelminenspital, Montleartstrasse 37, 1160 Vienna, Austria. E-mail: daniel.dobererameduniwien.ac.at

ABSTRACT Asthma, and severe asthma, in particular, is increasingly recognised as a heterogeneous disease. Identifying these different phenotypes of asthma and assigning patients to phenotype-specific treatments is one of the current conundrums in respiratory medicine. Any diagnostic procedure in severe asthma (or any disease) should have two aims: 1) better understanding or identifying the diagnosis, and 2) providing information on the heterogeneity of asthma phenotypes to guide therapy with the objective of improving outcomes. Lung biopsies can target the large and small airways as well as the lung parenchyma. All compartments are affected in severe asthma; however, knowledge on the distal lung is limited. At this point, it remains uncertain whether lung specimens routinely add diagnostic information that is unable to be obtained otherwise. Indeed, whether a lung biopsy is indicated in the workup of a patient with severe asthma remains an individual decision. It is hoped this review will support rational decision-making and provide a detailed synopsis of the varied histopathological features seen in biopsies of patients with a diagnosis of severe asthma. Due to limited data on this topic this review is primarily based on opinion with recommendations arising primarily from the personal experience of the authors.

@ERSpublications

Optimising use of lung biopsies in a diagnostic workup of severe asthma requires a multidisciplinary approach http://ow.ly/PdLW2

\section{Introduction}

Asthma is a clinical diagnosis based on symptoms, detailed history and pulmonary function testing [1]. It is one of the most common and longest described respiratory diseases, but remains elusive and poorly defined. It has long been appreciated that asthma is an umbrella term for patients meeting certain clinical and physiological criteria, but who differ on the basis of specific clinical, biological and/or therapeutic characteristics [2]. Thus, it is difficult to define the pathology of a condition that probably, at a minimum, represents a spectrum of biological processes, and perhaps, even different diseases [3]. The pathological

Previous articles in this series: No. 1: Dombret M-C, Alagha K, Boulet LP, et al. Bronchial thermoplasty: a new therapeutic option for the treatment of severe, uncontrolled asthma in adults. Eur Respir Rev 2014; 23: 510-518. No. 2: Barnig C, Levy BD. Innate immunity is a key factor for the resolution of inflammation in asthma. Eur Respir Rev 2015; 24: 141-153. No. 3: Papaioannou AI, Kostikas K, Zervas E, et al. Control of asthma in real life: still a valuable goal? Eur Respir Rev 2015; 24: 361-369.

Received: May 262015 | Accepted after revision: June 132015

Conflict of interest: Disclosures can be found alongside the online version of this article at err.ersjournals.com

Provenance: Submitted article, peer reviewed.

Copyright OERS 2015. ERR articles are open access and distributed under the terms of the Creative Commons Attribution Non-Commercial Licence 4.0. 
understanding is further complicated by the influences of disease severity, the complexities of the lung itself and the associated heterogeneity of pathological changes throughout the bronchial tree.

\section{Different lung compartments involved in asthmals)}

Traditionally, asthma has been regarded as a disease characterised by chronic inflammation and remodelling, primarily of the large airways, which leads to airflow obstruction and asthma-specific symptoms. Over 100 years ago, autopsy specimens revealed macroscopic pathology within the large airways [4]. However, subsequent physiological, imaging and pathological studies demonstrated that the small airways and even the lung parenchyma could be important contributors to the functional impairment seen in certain severe asthma patients, as some reports suggest asthma severity increases in proportion to the involvement of this compartment $[5,6]$. Small airways include all peripheral membranous bronchioles $<2 \mathrm{~mm}$ in diameter, whereas large airways contain smooth muscle and cartilage and have a luminal diameter $>2 \mathrm{~mm}$ (bronchi up to terminal bronchioles) [7]. Numerous reviews on the importance of small airways in asthma and other airway diseases have been published in recent years, including the following [8-12].

Clinical symptoms and physiological assessment are not able to accurately predict dysfunction in small airways, probably because distal airways normally contribute $<10 \%$ of the total resistance to airflow $[7,13]$. Hence, Hogg and colleagues, who also first used the term "small airway disease" [7], described the distal airways as the "silent zone" of the airway tree as extensive disease can be present without symptoms or changes in conventional lung function tests [14]. In the 1970s, the first physiological studies using indirect measurements of the distal airways (primarily nitrogen washout) focused attention on the role of small airways in diseases like asthma and chronic obstructive pulmonary disease [15-17]. Since then, more sophisticated invasive and noninvasive techniques for measurements of the distal airways have supported the importance of distal airway resistance $[18,19]$. In addition, evidence of a low diffusing capacity of the lung for carbon monoxide (DLCO), which is typically reduced in patients with interstitial lung disease (ILD), but traditionally normal or high in asthma [20], may serve a marker of distal lung involvement or remodelling in some patients with severe (and perhaps atypical) forms of asthma [21].

The small airways are also beyond the current imaging resolution of high-resolution computed tomography (HRCT) scanners, but "indirect signs" of the effects of small airway disease on the lung parenchyma can be detected as well as "direct signs" like centrilobular nodules or "tree in bud" patterns. Air trapping and heterogeneity of lung ventilation due to small airway disease correlate with asthma severity and adverse outcome [22, 23]. While changes in airway wall thickness in the large airways have been reported to be reduced in response to anti-eosinophilic (interleukin (IL)-5) therapies [24] and inhaled corticosteroids (ICS) [25], HRCT imaging features associated with distal lung changes have not yet been linked to molecular phenotypes or responses to therapy [26].

Early autopsy pathological studies which evaluated the total lung demonstrated that the entire length of the bronchial tree is involved in patients dying of asthma [27-29]. Comparison with milder asthma patients dying of other causes was often limited, but generally showed less inflammation and remodelling in the distal lung than those who died of asthma [27]. Importantly, these studies were done before the widespread use of ICS, which may have impacted the findings, given their association with a reduction in asthma mortality [30]. Studies of the distal lung in living asthma patients are extremely limited. Resected lung specimens from asthmatic patients undergoing thoracic surgery demonstrated that the inflammatory response is not restricted to the proximal airways, although very minimal clinical information on these patients was available [21, 31-33].

Transbronchial biopsy (TBBx) studies have suggested that the intensity of inflammation may be higher in the distal compared with the central airways in patients with asthma treated with high doses of inhaled and even systemic corticosteroids [34]. Kraft and colleagues found increased eosinophilic and lymphocytic inflammation in distal airways and alveolar spaces from TBBx at 04:00 h in asthma patients with nocturnal awakenings when compared with endobronchial biopsies (EBBx) [35, 36]. Similar results were shown for mast cells, where alveolar mast cell density was increased in asthmatic patients but bronchial mast cell density was not $[37,38]$. Additional studies suggested that this "classical/allergic" inflammatory pattern may not be present in the distal lung of all asthmatics, with a predominance of neutrophils over eosinophils in the bronchoalveolar lavage (BAL) fluid, and endobronchial and transbronchial samples in some severe asthma patients on high-dose corticosteroids [39, 40]. BAL neutrophils have also been associated with air trapping on quantitative HRCT imaging [23], potentially linking the neutrophilic phenotype to the distal lung compartment.

These distal lung studies began to link distal airway inflammation with specific symptoms and disease severities. While these studies suggest that small airways and even alveolar tissue may be crucial compartments of the lung which determine disease severity, clinical symptoms and even phenotypes of asthma, their utility 
in guiding clinical management of patients is unclear. In addition, it remains unknown whether distal airway involvement is present in all (severe) asthma patients or whether a "distal airway phenotype" exists.

\section{Severe asthma is not a single disease}

Although asthma has long been considered an eosinophilic disease, as early as the 1950s it was recognised that eosinophilic inflammation was not present in all asthma patients and that those without eosinophilic inflammation responded less well to corticosteroid therapy [41]. In the 1980s and 1990s, the introduction of relatively safe and effective ICS led to a "one size fits all" approach to asthma and the conclusion that these drugs, alone or in combination with long-acting $\beta$-agonists, could effectively treat nearly all patients. Thus, the utility of understanding underlying differences in pathobiology became less. However, by the late 1990s it was recognised that a subgroup of patients with more severe disease did not adequately respond to this approach and remained highly symptomatic with reduced lung function [40,42]. These concepts were further solidified by the inability of targeted biological therapies to be effective in either specific subtypes of asthma (allergen-responsive) or all comers with asthma [43,44]. Thus, by the late 1990s, the concept of asthma phenotyping was beginning to re-emerge, particularly in those patients with severe asthma. Initially, severe asthma patients were divided into those with and without tissue eosinophilia [40, 45], and later by using less-biased statistical clustering methods [24, 46-49]. The field further advanced when the concept of molecular phenotyping identified patients with a T-helper cell (Th)2 molecular signature in airway epithelial cells [50]. This Th2 phenotype is now referred as the Type 2 signature given the range of cells producing IL-4, -5 , and -13. Several Type 2 cytokine-related biomarkers (table 1) have been identified and used to predict the response to treatment with monoclonal antibodies to IL-5, IL-13 and IL-4 receptors. $~ 50 \%$ of severe asthma patients express a Type 2 signature. It appears that these less invasive biomarkers may adequately identify moderate-to-severe patients for these Type 2-targeted approaches. However, it remains to be determined whether additional benefit will be obtained by identifying tissue histopathology in more severe patients with persistent elevations in exhaled nitric oxide fraction ( $F$ eNO) or blood/sputum eosinophils, despite high doses of systemic corticosteroids or whether these patients will respond similarly to Type 2-directed therapies. Two recent studies suggest that not all patients with biomarker evidence for Type 2-associated asthma, especially in very severe disease, will respond to Type 2-targeted therapies (both anti-IL-5 and anti-IL-13) [51, 52]. Reasons for these poor responses to targeted therapies may relate to underlying differences in driving the more complex pathobiological features not currently addressed by these biomarkers.

Little is understood about "Type 2-low" asthma in terms of pathobiology. Some studies have suggested isolated neutrophilic inflammation and differences in structural characteristics, e.g. subepithelial basement membrane thickness $[45,53]$. However, almost nothing has been described regarding pathology (and certainly distal lung pathology) in these Type 2-low patients, even those with high levels of symptoms and abnormal physiology.

\section{A clinical-radiological-pathological approach to severe asthma}

Typically, evaluation of patients with difficult/severe asthma includes a comprehensive history, physical examination, pulmonary function testing, and laboratory evaluation including a complete blood count and differential, immunoglobulins, IgE and specific allergen testing. A multidisciplinary and multiprofessional approach, including involvement of related medical specialities, respiratory nurses, physiotherapists, psychological consultants and coordinated interactions of the different caregivers, is also critical $[54,55]$.

Chest radiographs, specifically HRCT scans, are carried out by many physicians to rule out comorbid illness, with the European Respiratory Society (ERS)/American Thoracic Society (ATS) guidelines suggesting they could be performed in patients with atypical presentations of asthma [56]. Although the increased spatial resolution of HRCT allows noninvasive assessment of remodelling, its utility in diagnosing molecular phenotypes of asthma or even different airway diseases remains poorly quantified [26, 57-59]. The use of more invasive studies, in particular lung biopsies, in severe asthma is even more controversial.

However, a clinical-radiological-pathological approach is well established in other fields of respiratory medicine, e.g. oncology or ILDs. Even more than the syndrome of "asthma", ILD includes a heterogeneous

TABLE 1 Biomarkers of Type 2 inflammation in severe asthma

Exhaled nitric oxide fraction

Increases in total IgE

Serum periostin

Sputum/blood eosinophilia

Sputum expression of interleukin-4, -5 and -13 
group of lung pathologies, with different aetiologies, ranging from inflammatory disorders to progressive fibrotic conditions. Although ILD can be challenging to diagnose, consensus statements have established criteria to define clinical-radiological-pathological entities that differ in pathogenesis, treatment strategy and prognosis [60]. None of the involved disciplines, i.e. the clinician, radiologist and pathologist, may be able to provide a reliable diagnosis but close multidisciplinary communication leads to better results.

Differential diagnosis of systemic corticosteroid treated "asthma"

Several conditions may mimic or be associated with severe asthma in which surgical lung biopsies might be useful for differentiation (table 2).

\section{Eosinophilic granulomatosis with polyangiitis}

Eosinophilic granulomatosis with polyangiitis (EGPA or Churg-Strauss syndrome) is a multisystem vasculitis of the small- and medium-sized arteries characterised by chronic sinusitis, severe asthma symptoms, parenchymal infiltrates and prominent peripheral blood eosinophilia. However, in the face of systemic corticosteroids, peripheral blood eosinophilia may be minimal. Therefore, it can be challenging to differentiate severe asthma from EGPA, especially as vasculitis is often not apparent in the initial (prodromal) phase of EGPA [61]. However, the presence of extrapulmonary vasculitis or symptoms, e.g. in the skin, gastrointestinal, renal or central nervous system, would not be consistent with severe asthma [61].

\section{Hypersensitivity pneumonitis}

Hypersensitivity pneumonitis (HSP) is, like asthma, a complex syndrome and not a single, uniform disease. There is no clinical, laboratory, imaging or other "gold standard" for the diagnosis or classification into acute, subacute and chronic HSP. BAL and TBBx are helpful in supporting the diagnosis of HSP. However, in many instances surgical biopsy is indicated, particularly in cases where no offending antigen is identified or more advanced disease is present [62].

\section{Asthmatic granulomatosis}

The term asthmatic granulomatosis was introduced in 2012 [21] for patients meeting the criteria of severe asthma but with atypical histopathological findings of interstitial and bronchial mononuclear infiltrates with poorly formed non-necrotising granulomas. This histopathological pattern might suggest subacute or chronic HSP but, in addition, these cases presented with asthmatic (often eosinophilic) small airway changes that are not part of the spectrum of HSP.

\section{Autoimmune airway disease}

The involvement of the lungs in systemic autoimmune diseases is common and ranges from $~ 5 \%$ in rheumatoid arthritis or systemic lupus erythematosus [63], to $40-60 \%$ in inflammatory bowel disease [64], and up to $90 \%$ in anti-neutrophil cytoplasmic antibodies-associated vasculitides [65]. Despite the strong association of connective tissue disease with ILDs, information on the involvement of small airways (bronchiolitis) is limited. Several patterns have been recognised, in particular in rheumatoid arthritis and Sjögren's syndrome [66, 67], e.g. cellular or follicular bronchiolitis involving lymphocytes, neutrophils and macrophages. However, they are not normally linked to reversible airflow limitation associated with "asthma".

\section{Allergic bronchopulmonary aspergillosis}

Allergic bronchopulmonary aspergillosis (ABPA) is a complex hypersensitivity reaction occurring when bronchi become colonised by Aspergillus species in patients with asthma or cystic fibrosis. Clinically, ABPA presents with asthma-like symptoms complicated by recurrent episodes of fever, malaise, expectoration of

\section{TABLE 2 Differential diagnoses of systemic corticosteroid treated "severe asthma"}

Eosinophilic granulomatosis with polyangiitis (Churg-Strauss syndrome) ${ }^{\#}$

Hypersensitivity pneumonitis (including hot tub lung)

Chronic aspiration ${ }^{\#}$

Sarcoidosis $\#$

Allergic bronchopulmonary mycosis

Constrictive bronchiolitis ${ }^{\#}$

Asthmatic granulomatosis ${ }^{\#}$

Autoimmune airway disease ${ }^{\#}$

Infectious diseases (atypical bacteria, fungal or nontuberculous mycobacteria)

\footnotetext{
\#: diseases where lung biopsies might be helpful in the diagnostic evaluation.
} 
brownish/green mucus and, sometimes, haemoptysis. In addition to pathological features of asthma [68], bronchocentric granulomatosis, lymphocytic infiltrates, septated hyphae with acute dichotomous branching in the mucus-filled bronchial lumen or positive cultures for Aspergillus may be seen in ABPA. Unlike the other diseases, the diagnosis of ABPA is not based on pathology, but is confirmed by use of clinical, radiological and immunological criteria $[68,69]$.

\section{Lung biopsy in patients with severe asthmals)}

Whether invasive studies, particularly bronchoscopy with biopsy, lavage, or both, or surgical biopsy are indicated in severe asthma is not generally agreed upon. However, numerous studies suggest that pathological changes exist in the small airways and lung parenchyma of subjects with (severe) asthma [21, 27, 28, 31-37], including the presence or absence of certain patterns of inflammation, colonisation/ infection with atypical bacteria or fungi, or both, which might support changes in therapy. In addition, certainly in children, but also in adults, visual evaluation of the airways is often considered necessary to rule out structural abnormalities, aspiration or infection. Therefore, in many patients with severe asthma the diagnostic workup will already include a bronchoscopic procedure. However, the importance of bronchoscopic or surgical biopsies to diagnose, phenotype and treat "severe asthma" is more controversial.

\section{Who is a candidate for lung biopsy?}

Severe asthma represents a spectrum of disease that remains difficult to control, and therefore, causes high morbidity and poses a massive burden on healthcare [70-73]. Patients with severe asthma have complex treatment requirements, which in $30-40 \%$ of cases include the continuous use of systemic corticosteroids $[74,75]$. The high risk-benefit ratio of such systemic therapy can result in serious and often irreversible adverse effects $[76,77]$, such that means to avoid long-term systemic corticosteroid use in severe asthma should be considered [56]. At the University of Pittsburgh Asthma Institute (Pittsburgh, PA, USA) only a minority $(\sim 30 \%)$ of patients with very severe, systemic corticosteroid-dependent asthma undergo clinically indicated lung biopsies. Before lung biopsies are considered, patients referred with the diagnosis of "asthma" are optimised for diagnosis and treatment for several months, as recommended by the ERS/ATS severe asthma guidelines [56]. Prior to consideration of lung biopsy it is critical to address poor adherence in these patients, as it may be present in up to $60 \%$ of uncontrolled asthma $[78,79]$. Treatment adherence and misuse of inhalers are major determinants of outcomes/disease control in asthma [80,81], and this is particularly true for severe asthma patients requiring long-term treatment with high-dose inhaled and/or systemic corticosteroids [82]. Patients undergoing (surgical) lung biopsies should probably be embedded in a multi-professional team setting (discussed earlier), where these issues can be adequately addressed before serious consideration of biopsy.

Selection of patients for lung biopsy should address two points: 1) accurate diagnosis of the severe airway disease and/or 2) heterogeneity of severe asthma phenotypes to direct treatments. Therefore, patients with "atypical" features of severe, systemic corticosteroid-dependent asthma may be considered appropriate candidates for lung biopsies (table 3). Atypical features include findings suggestive of parenchymal disease, e.g. reduced DLCO [21] or parenchymal alterations in the HRCT.

Similarly, patients with consistently high FeNO or blood eosinophils [21], despite high corticosteroid doses, represent a poorly understood group who might qualify for more invasive diagnostics, especially since EGPA is part of the differential. Patients with severe asthma and a background of autoimmune disease, e.g. personal autoimmune disease, positive autoantibodies or family history of autoimmune disease, with less "allergic" history might also be considered. Pathologically those patients seem to be characterised by a predominant lymphocytic inflammatory pattern and appear to respond to immunosuppressive drugs.

\section{Pathological approaches}

In airway disease, unlike parenchymal lung diseases, the primary approach to obtaining pathological specimens has been through EBBx performed during bronchoscopy. Surgical or distal lung biopsies are exceedingly rare.

\section{Bronchoscopy}

Unlike the less invasive sputum or exhaled breath analyses, bronchoscopy can obtain lung tissue, cells and diluted fluids (in the form of BAL). EBBx of the large airways visible through the bronchoscope is the standard approach, with TBBx addressing distal airways plus lung parenchyma only rarely performed. Samples to be collected include cell counts (eosinophils, neutrophils, CD4/CD8 ratio, etc.), microbiological testing (bacteria, fungi and Mycobacteria), and tissue analysis. Visual airway inspection can add information on possible diagnoses, e.g. vocal cord dysfunction, gastro-oesophageal reflux disease, stenosis of large airways, tracheomalacia, tumour or foreign-bodies [85]. 
TABLE 3 Preoperative evaluation including indications and risk factors

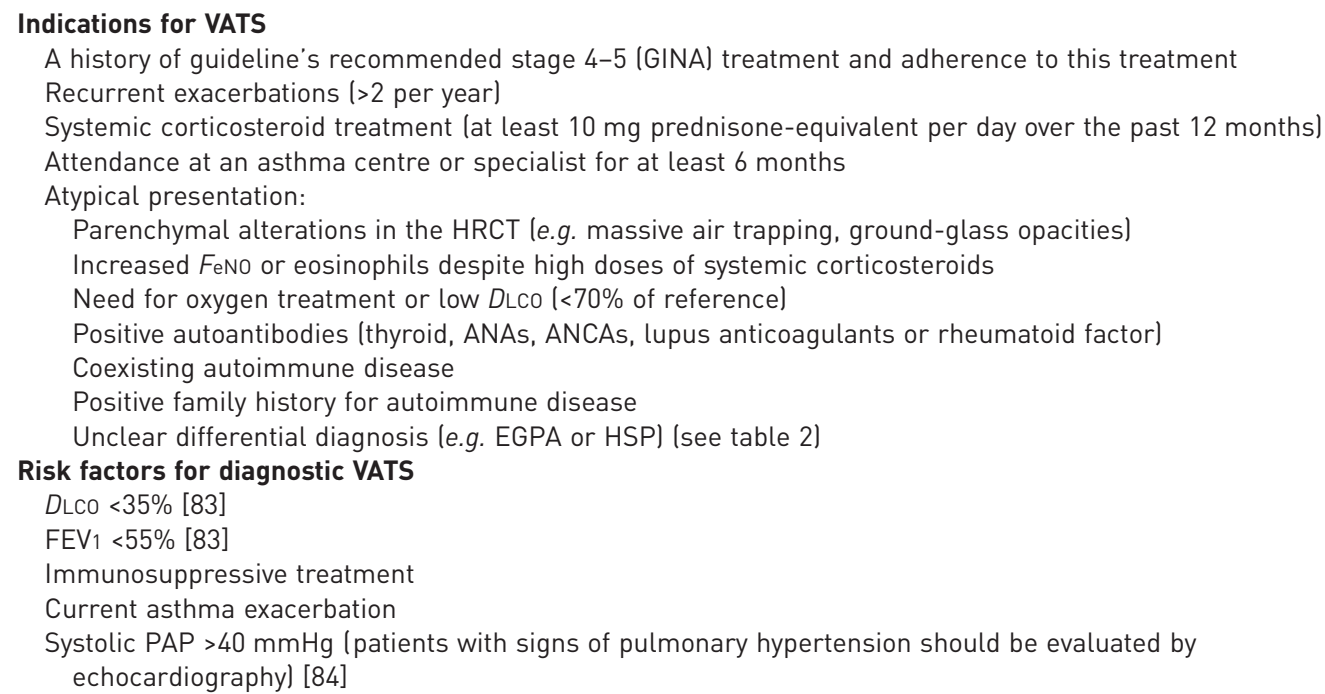

VATS: video-assisted thoracoscopic surgery; GINA: Global Initiative for Asthma; HRCT: high-resolution computed tomography; $F$ eNO: exhaled nitric oxide fraction; $D\llcorner C O$ : diffusing capacity of the lung for carbon monoxide; ANA: anti-nuclear antibodies; ANCA: anti-neutrophil cytoplasmic antibodies; EGPA: eosinophilic granulomatosis with polyangiitis; HSP: hypersensitivity pneumonitis; FEV1: forced expiratory volume in $1 \mathrm{~s}$; PAP: pulmonary arterial pressure.

EBBx specimens are taken from the main carina and subcarinae of the lower lobes and fixed in formalin, acetone or another preservative, or frozen. However, the samples obtained are extremely small (usually $1 \mathrm{~mm}^{3}$ or less), and represent a minute fraction of the total lung pathology; therefore, it is useful to take biopsies in different lobes, e.g. three biopsies in the right upper and lower lobe. Topical anaesthesia before and during bronchoscopy, e.g. lidocaine, is helpful and safe (up to $7 \mathrm{mg} \cdot \mathrm{kg}^{-1}$ lidocaine) to decrease coughing and the bronchoconstriction reflex $[86,87]$. The procedure itself should only be carried out by a highly experienced bronchoscopist with appropriate technical and nursing support. Bronchoscopy is commonly carried out under general anaesthesia in children, whereas in adults it is usually carried out using conscious sedation.

Although concerns have been raised about multiple bronchial biopsies in asthma patients [88], experience has demonstrated that up to $10 \mathrm{EBBx}$ can be obtained safely [89]. In addition, TBBx (up to four per patient under fluoroscopic guidance) have been safely performed in asthmatic patients $[34,36]$. Finally, the safety of bronchoscopy in adult patients with severe and very severe asthma (forced expiratory volume in $1 \mathrm{~s}$ (FEV 1 ) $<60 \%$ predicted) in the Severe Asthma Research Program [90] and in children [91] appears to be acceptable. Bronchoscopy has been used to phenotype asthmatic patients and was reported to successfully direct therapy [85]. Good et al. [85] defined four specific phenotypes based on bronchoscopic evaluation including visual inspection of the upper and lower airways as well as BAL, EBBx and brushes. 4 months of phenotype-adjusted (non-blinded or randomised) treatment was reported to improve asthma control and lung function (FEV1).

Readdressing the two important aims for lung biopsy of 1) diagnosing or ruling in/out asthma and 2) obtaining information on disease phenotypes to direct therapy, bronchoscopy itself appears to have a role as a diagnostic tool in severe asthma. However, bronchoscopic biopsies may have more limited value. These biopsies are subject to crush artefacts, with TBBx of limited value for small airways/parenchymal abnormalities, vasculitis, rare granulomas or inflammatory patterns. In addition, specific pathological diagnostic parameters are limited, often resulting in pathology reports with vague descriptions such as "scattered eosinophils", "no tumour seen", or "changes concordant with clinical asthma diagnosis". Although bronchoscopic biopsies might reveal more information, most pathologists are not comfortable in addressing subtle findings, especially in EBBx. Thus, further studies, with better pathological qualification and quantification will be required before bronchoscopic biopsies become routine procedures in patients with a severe asthma diagnosis.

\section{Surgical lung biopsies}

Peripheral lung tissue can be collected by open thoracotomy or video-assisted thoracoscopic surgery (VATS). Today, the minimally invasive VATS is preferred due to improved mortality, morbidity, post-surgical pain and duration of hospital stay compared with open thoracotomy [92]. Multiple locations of the lung can be 
accessed. VATS biopsies have become the gold standard to evaluate the distal lung, including small airways, vessels, peribronchovascular and alveolar space, and give access to samples of several millimetres (even centimetres) in size. Thus, VATS biopsies are more suitable to address pathologies involving the distal lung with regard to differential diagnosis of refractory "asthmas".

\section{Video-assisted thoracoscopic surgery}

VATS has almost never been performed in patients with refractory airway disease, especially those with elements of an "asthma-like" condition. In fact, the majority of VATS procedures are performed for diagnostic or therapeutic reasons in pulmonary nodules and for diagnostics in ILD. For most ILD patients, VATS is the preferred procedure to collect lung biopsies with some exceptions like sarcoidosis, organising pneumonia, eosinophilic pneumonia or hypersensitivity pneumonitis where bronchoscopic biopsies may be sufficient $[60,93]$. While the reasons for VATS rarely being used in "severe asthma" probably include the perception that ILD is a more severe disease than "asthma" and the lack of consistent HRCT changes for the surgeon to target, a surgical biopsy approach may still be helpful in both diagnosis and management of severe asthma. In the case of ILD, HRCT patterns are often helpful to select optimal sites for VATS biopsies [94]. While in severe asthma HRCT imaging might reveal airway wall thickening or bronchiectasis only [95], more atypical findings like ground-glass opacities, extensive small airway disease or fibrosis might suggest further invasive evaluation (table 3 ).

\section{Preparation for VATS}

As VATS biopsies, like any surgical procedure, are not without risk, a risk-benefit assessment is important and must be discussed in detail with the patient. VATS should be performed in centres with experience of severely compromised pulmonary patients, e.g. lung transplant centres. Prior to VATS all patients should undergo: 1) risk evaluation, 2) pre-surgical asthma treatment optimisation, and 3) selection of the biopsy site.

Patients with severe asthma undergoing VATS should only be those who require high-dose systemic corticosteroids to maintain control of their disease. However, this treatment may mask histopathological findings in the biopsies. Therefore, the corticosteroid dose is carefully decreased before surgery (for at least 1-2 weeks) to a level where the procedure is safe to perform, but there is modest evidence for inflammatory or physiological decline, as evidenced by increased blood eosinophils or FeNO, or decreased spirometry or DLCO measurements. The concern is that if the patient is asymptomatic and on a high systemic corticosteroid dose, the pathology may reflect the corticosteroid effect (decreased eosinophilic and other inflammatory processes) to the exclusion of the underlying pathology.

In ILDs the region of lung biopsy is selected for each patient in an agreement between the surgeon, clinician and radiologist, but also by intra-operative appearances and finger palpation. Because many severe asthma patients have minimal radiological abnormalities by HRCT, biopsies often have to be taken randomly. However, ground-glass opacities/inflammation or signs of small airway disease on HCRT can be a guide to select areas of interest. If no specific finding is present in a bilateral disease such as asthma, many surgeons prefer the right-sided approach as it provides easier access to biopsies at a lung edge.

\section{The VATS procedure}

Prior to surgery, patients are routinely treated with stress (or higher) doses of systemic corticosteroids, as well as bronchodilators. A standard VATS procedure is carried out under general anaesthesia using a three-port technique with the port sites placed to target the site of interest. Double-lumen endotracheal intubation with single-lung ventilation is used and the duration of the procedure is usually $<1 \mathrm{~h}$. The cone excision technique is preferred to yield resections of a generous size including deep tissue [21]. Usually, the procedure includes a preceding bronchoscopy to secure the position of the endotracheal tube and for visualisation of any endobronchial pathology in the mainstem bronchi. Additional sampling is rarely performed (e.g. BAL or brushes on the opposite side to the subsequent VATS biopsy). When the main differential diagnosis is inflammatory disease related, intra-operative frozen sections are not necessary. Samples should generally be obtained from each lobe of the targeted lung, especially when no targeted sites are identified. In addition to the histopathology, samples for fungal, bacterial and mycobacterial cultures should be collected.

Post-VATS care

Many patients can be safely discharged after 1 or 2 days of hospitalisation. Asthma treatment is re-established according to the pre-surgical treatment, in particular the systemic corticosteroid dose and bronchodilator treatment. 
The risks of a VATS procedure

There are few data on the risk of a VATS procedure in severe asthma. However, in our experience of about 50 airway disease VATS procedures, there was no increased risk of post-VATS asthma exacerbations, and in general, post-VATS surgical risk appears similar to those for other indications. In other diseases, the surgical morbidity and nonlethal complication rate is $7-10 \%$, with pulmonary fistula/pneumothorax, prolonged air leak, pleural effusion and acute exacerbation of the underlying disease (ILD) the most common complications $[94,96]$. Over the past 8 years, 51 VATS procedures in 47 "severe asthma" patients (46 adults and one child, 40 females and seven males, among whom four patients received a repeated VATS procedure due to lung nodules/masses) have been performed at our centre. The 30 -day mortality was $0 \%$. Nonlethal complications appeared in five patients (10\%): one haemothorax with two surgical revisions (22 days of hospitalisation), one wound healing issue needing a vacuum-assisted closure system, one post-surgical air leak with subcutaneous emphysema and two post-surgical pain syndrome needing re-hospitalisation. Only four (8\%) patients needed post-surgical intensive care. $\sim 40 \%$ of patients were discharged the next day after the VATS biopsy (the median duration of post-surgical hospitalisation was 2.0 days).

There are no clear contraindications to a VATS. Table 3 provides a list of factors that are usually associated with increased risk when undergoing a diagnostic VATS (for ILDs) [83]. Typical preoperative screening should include an ECG, in addition to tests already obtained in the evaluation of these "atypical" severe asthma patients like HRCT and lung function testing. Patients with suspected pulmonary hypertension should be evaluated by echocardiography because a systolic pulmonary arterial pressure $>40 \mathrm{mmHg}$ is a known risk factor for VATS-associated complications [84]. While asthma is not traditionally a risk factor for pulmonary hypertension [97], there are no reliable data on atypical severe asthma, in particular those with atypical findings like autoimmune comorbidities, hypoxia or reduced DLCO.

\section{Pathological evaluation of peripheral lung tissue in patients with "severe asthma"}

The histopathology of asthma was first described from autopsy studies of individuals dying of asthma. These studies demonstrated changes in large and small airways including mucus plugging, epithelial detachment, goblet cell metaplasia, basement membrane thickening, subepithelial fibrosis, and smooth muscle and mucous gland hypertrophy [98-100]. Nearly all inflammatory cells have been linked to asthma, including eosinophils, lymphocytes and mast cells/basophils as well as neutrophils, often in varying proportions. Individual histological features are neither specific nor sensitive for asthma, but a combination of several of the features generally suggests a diagnosis of what has traditionally been termed "asthma".

As in asthma, severe asthma lung tissue should be evaluated for both inflammatory and structural/ remodelling elements [100]. The following synopsis on classical as well as atypical histopathological findings in "severe asthma" includes information on small airways and lung parenchyma. A surgical lung biopsy in patients with atypical "severe asthma" should include histopathological analyses including haematoxylin and eosin stains, special stains for fungi and acid-fast bacilli (AFB), and immunohistochemistry (IHC) as well as microbiological testing with cultures for mycobacteria and fungi. Few institutions and pathologists have experience with surgical lung specimens from asthmatic patients and evaluation of such tissue should be limited to experienced centres.

\section{Structural changes and remodelling Epithelium}

Goblet cell hyperplasia/metaplasia is the most significant finding identified in the large airway epithelium, in particular in severe asthma [101-103]. Goblet cells produce mucin glycoproteins (MUC), with MUC5AC being predominant in asthmatic airways in response to Type 2 cytokines [104]. In cases of fatal asthma, there is often extensive luminal mucus plugging in both large and small airways, frequently mixed with inflammatory cells $[105,106]$. In distal lung biopsies from living asthma patients, goblet cell metaplasia and mucus plugging are present but may not be pronounced [21]. The relevance of epithelial shedding in EBBx may reflect tissue sampling artefacts [107], while in surgical biopsies it is more likely to reflect an abnormal epithelium (fig. 1).

\section{Subepithelial fibrosis}

Subepithelial fibrosis is a striking finding in the large and small airways of severe asthma. It involves two components: 1) reticular basement membrane (RBM) thickening and 2) submucosal fibrosis. The basement membrane consists of a superficial layer (the basal lamina) and a deeper layer (the lamina reticularis, or RBM). In asthma, thickening is restricted to the RBM. It has been extensively studied as a marker of remodelling in asthma and severe asthma, but the reported studies are conflicting. Some investigators have concluded that a thickened RBM is the most discriminative histological finding of severe asthma, generally not occurring in other chronic obstructive lung diseases [108, 109], whereas others have suggested that it may be unrelated to asthma severity [110]. An eosinophilic or Type 2 cytokine-associated 

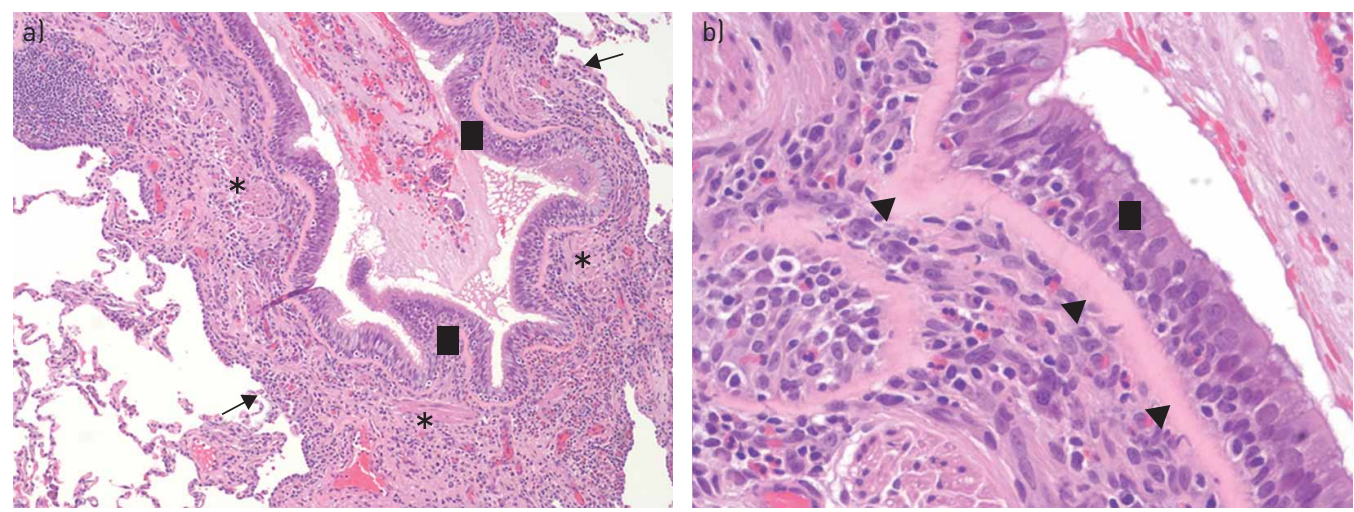

FIGURE 1. Examples of "classical" small airway disease in severe asthma from video-assisted thoracoscopic surgery procured tissue showing small airways with epithelial hyperplasia and mucous plugs (black squares), prominent smooth muscle hyperplasia/hypertrophy (asterisks), thickened basement membrane (arrowheads) and adventitial eosinophilic/lymphocytic inflammation extending into areas of alveolar attachments (arrows). a) 100× magnification, b) 400× magnification.

process appears to be critical for RBM thickening [40, 111]. In our centre, RBM thickening in small airways was present in all patients with asthmatic granulomatosis and was marked in $50 \%$ of them [21]. Remodelling of the submucosal extracellular matrix in severe asthma is much less characterised (fig. 1).

Increased airway smooth muscle mass

It is believed large airway smooth muscle thickening in asthma is a consequence of both hypertrophy (increased size) and hyperplasia (increased proliferation) of airway smooth muscle cells [112], both of which correlate with asthma severity [113, 114], but not with age or duration of disease [112]. In small airways, increased smooth muscle cell mass was seen in severe asthma patients with asthmatic granulomatosis; however, it may not be specific to any severe asthma phenotype [21] (fig. 1).

\section{Inflammatory patterns}

Inflammatory patterns are probably more important indicators for disease severity, phenotypes or differential diagnoses than structural changes or remodelling. Inflammatory cells can be assessed qualitatively or (semi)-quantitatively (cells per area). For a quantitative approach the small airway wall is usually divided into different areas of interest $[5,31,115,116]$ : lumen, epithelium, inner airway wall (between the basement membrane and the smooth muscle), smooth muscle, outer airway wall (between the smooth muscle and the alveolar attachment), the alveolar attachment, and peripheral alveolar tissue (fig. 2). Quantitative analysis is limited to research because it is time consuming and reference values are not yet available.

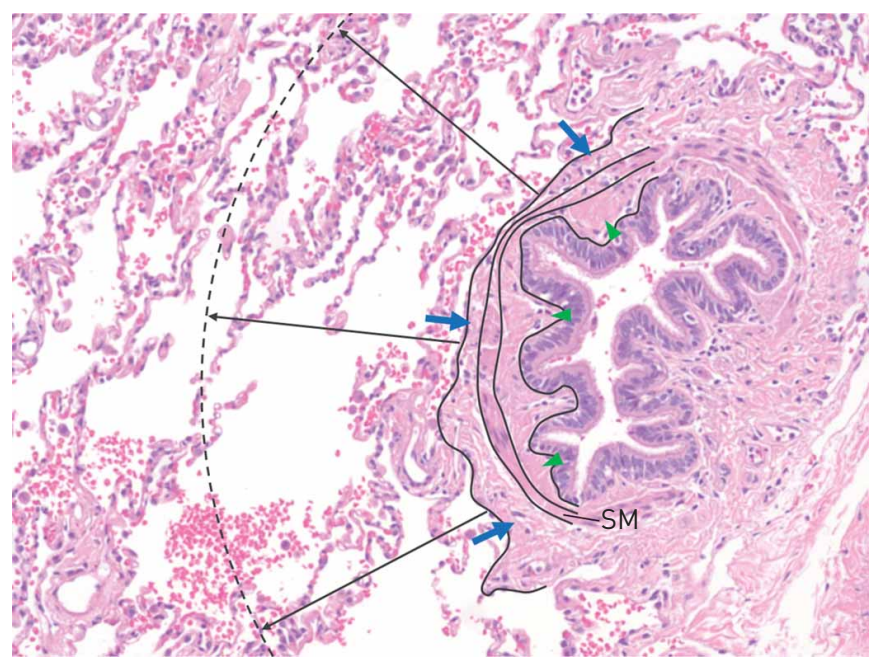

FIGURE 2. Outline of the distal lung regions in haematoxylin and eosin-stained lung tissue $[115,116]$ showing the inner airway wall (green arrowheads), smooth muscle (SM), outer airway wall (blue arrows) and alveolar attachment (black arrows). 
Eosinophilic inflammation

Early studies of resected lung tissue confirmed that asthmatic small airways have higher numbers of activated eosinophils than larger airways [31]. Likewise, eosinophils and macrophages can accumulate in the alveolar tissue of nocturnal asthmatics [35]. In large airways, eosinophils predominate in the inner airway wall, whereas in small airways they predominate in the outer airway wall [115, 117]. Examples of small airway eosinophilic inflammation in VATS tissue from severe asthma patients can be seen in figure 1. Eosinophilic inflammation is nonspecific and could be seen in EGPA, asthmatic granulomatosis and corticosteroid-resistant severe asthma. However, detailed comparisons of the amount or location of eosinophils in these diseases are lacking.

\section{Neutrophilic inflammation}

The "classical" eosinophilic inflammatory pattern may not be present in the distal lung [39] or large airways $[40,117,118]$ of all asthmatic patients, but neutrophils may predominate. However, we have not yet found this pattern in our distal lung biopsies.

\section{Non-necrotising granuloma and lymphocytic bronchiolitis}

Non-necrotising granuloma and lymphocytic bronchiolitis are additional inflammatory patterns recently described in patients with atypical "severe asthma" (fig. 3a, b) [21]. Airway (predominance in the outer airway wall) and patchy interstitial mononuclear infiltrates (mostly CD4 or CD8 positive cells) and/or poorly-formed interstitial non-necrotising granuloma were found. While it remains unclear whether these patterns define a certain "severe asthma" phenotype associated with autoimmune diseases or represent the pulmonary involvement in a systemic autoimmune disease, many of these patients have a personal or family history of autoimmunity [21]. Early data suggest patients with this pattern may benefit from a treatment strategy involving alternative immunosuppressive drugs, like azathioprine.
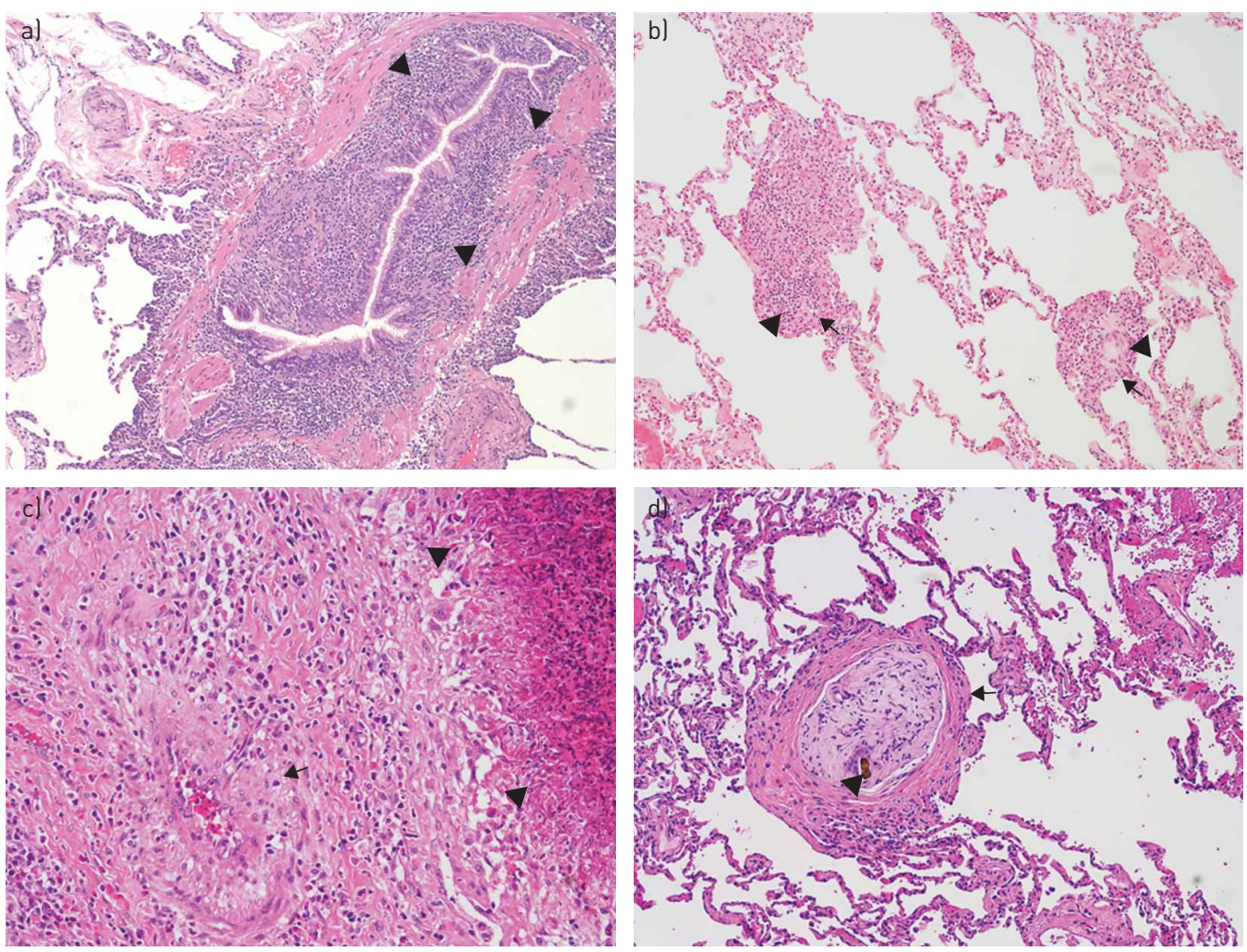

FIGURE 3. Examples of distal lung disease in "atypical" severe asthma and differential diagnoses from video-assisted thoracoscopic surgery procured tissue. a) Small airway with massive lymphocytic bronchiolitis (arrowheads) often found in severe asthma with an autoimmune background (100× magnification). b) Small interstitial poorly defined granulomas (arrows) with epitheloid histiocytes/giant cells (arrowheads) in asthmatic granulomatosis, note the absence of vasculitis (100× magnification). c) Granulomatous inflammation in eosinophilic granulomatosis with polyangiitis, note the presence of prominent tissue eosinophilia, vasculitis (arrow) and necrotising granulomatous inflammation (arrowheads) (200× magnification). d) Aspiration granuloma, note a well-formed, non-necrotising granuloma entirely replaces an airway lumen (arrow), with foreign-body giant cell reaction to vegetable material (arrowhead) (100× magnification). 


\section{Effects of corticosteroids}

All of our severe asthma patients undergoing lung biopsy, as well as those in the previously published report [21], were on daily oral corticosteroids (with/without high-dose ICS). EBBx studies in mild-to-moderate asthma have shown that ICS and oral corticosteroids decrease epithelial and submucosal eosinophils, mast cells and macrophages [119]. Oral corticosteroids may also increase mucosal neutrophils in mild-to-moderate non-atopic asthma [120]. Thus, oral corticosteroid treatment might explain the scarce eosinophils or mast cells seen in many of our severe asthma biopsies. However, increased neutrophils (in any compartment) were not observed.

\section{Immunohistochemistry and special stains}

IHC should be performed when there is marked inflammatory infiltrate to specifically rule out other entities like lymphoma [121]. IHC can also provide information on inflammatory patterns; however, there are no data or experience on the selection of the markers, or their impact on diagnosis, asthma phenotyping or prediction of treatment responses. Special stains (as well as cultures) to rule out fungal infections (e.g. Grocott's methenamine silver or periodic acid-Schiff stain) and AFB for mycobacteria should always be performed, especially in the presence of granulomatous inflammation.

\section{Other pathologies}

In patients with "severe asthma", VATS biopsies at our centre not only provided information on different "asthmatic" histopathological patterns, but not uncommonly also led to revision of the initial diagnosis. In three patients histopathology showed foreign body (food) granulomas (fig. 3d) although extensive previous evaluation did not implicate chronic aspiration. The diagnosis of sarcoidosis based on a bronchoscopic biopsy performed several years earlier was overturned based on a clinical presentation consistent with asthma in combination with a lung biopsy consistent with asthmatic granulomatosis. Although EGPA (a typical image is shown in fig. 3c) was in the differential diagnosis for many of our severe asthma cases, in fact, vasculitis was never seen in our VATS cohort. Finally, HSP (and not asthma-related disease) was found on an additional VATS from our centre.

\section{Importance of surgical lung biopsies in research}

Knowledge of the mechanisms and underlying pathobiology driving many of these chronic severe airway diseases and phenotypes is extremely limited. VATS samples could provide valuable resources for research. Immunohistochemical, protein and RNA analysis of resected lung tissue can provide important information to elucidate the pathobiological patterns of severe asthma. With the larger amount of VATS tissue available, additional approaches might include compartment-specific (microdissection) or automated quantitative digital pathology. Finally, it is critical that results from VATS biopsies are compared with results from less invasive bronchoscopies, new imaging techniques such as magnetic resonance imaging, noninvasive physiological markers of distal lung abnormalities (e.g. multiple-breath nitrogen washout tests), or other peripheral biomarkers different from the well-known Type 2-related ones with the aim of eventually predicting VATS findings (and avoiding the more risky procedure).

\section{Conclusion}

The decision for or against a lung biopsy has to be evaluated for each individual patient based on a riskbenefit assessment. The benefits of a surgical lung biopsy include a potential revision of a diagnosis and identification of disease phenotypes of "asthma" that may benefit from specific treatments, e.g. patients that might benefit from immunosuppressive drugs, antifungals, etc. The risks include the invasiveness of the procedure and the possibility that no helpful information will be gained. However, based on our experience, we believe VATS biopsies give a better opportunity to differentially diagnose very severe, corticosteroid-dependent phenotypes or conditions masquerading as this phenotype, and provide information which could successfully change therapy when compared with bronchoscopic biopsies (EBBx or TBBx). Optimising the use of lung biopsies in a diagnostic workup of severe asthma probably requires a multidisciplinary clinical-radiological-pathological approach and collaboration across multiple sites specialising in severe asthma. Further studies are needed in this area to fully ascertain their importance.

\section{References}

1 Global Initiative for Asthma. Global strategy for asthma management and prevention, revised 2014. Date last accessed: May 20, 2015. Date last updated: April 2015. www.ginasthma.org

2 Wenzel SE. Asthma phenotypes: the evolution from clinical to molecular approaches. Nat Med 2012; 18 716-725.

Wenzel SE. A plea to abandon asthma as a disease concept. Lancet 2006; 368: 705.

Cruschmann H. Ueber bronchiolitis exsudativa und ihr Verhaeltnis zum asthma nervosum. Dtsch Arch Klin Med 1882; 32: 1-34. 
Dolhnikoff M, da Silva LF, de Araujo BB, et al. The outer wall of small airways is a major site of remodeling in fatal asthma. J Allergy Clin Immunol 2009; 123: 1090-1097.

Wenzel SE, Busse WW. Severe asthma: lessons from the Severe Asthma Research Program. J Allergy Clin Immunol 2007; 119: 14-21.

$7 \quad$ Hogg JC, Macklem PT, Thurlbeck WM. Site and nature of airway obstruction in chronic obstructive lung disease. N Engl J Med 1968; 278: 1355-1360.

8 Hyde DM, Hamid Q, Irvin CG. Anatomy, pathology, and physiology of the tracheobronchial tree: emphasis on the distal airways. J Allergy Clin Immunol 2009; 124: Suppl., S72-S77.

9 van den Berge M, ten Hacken NH, Cohen J, et al. Small airway disease in asthma and COPD: clinical implications. Chest 2011; 139: 412-423.

10 Burgel PR, Bergeron A, de Blic J, et al. Small airways diseases, excluding asthma and COPD: an overview. Eur Respir Rev 2013; 22: 131-147.

11 Bonini M, Usmani OS. The role of the small airways in the pathophysiology of asthma and chronic obstructive pulmonary disease. Ther Adv Respir Dis 2015 [In press DOI: 10.1177/1753465815588064].

12 van der Wiel E, ten Hacken NH, Postma DS, et al. Small-airways dysfunction associates with respiratory symptoms and clinical features of asthma: a systematic review. J Allergy Clin Immunol 2013; 131: 646-657.

13 Macklem PT, Mead J. Resistance of central and peripheral airways measured by a retrograde catheter. $J$ Appl Physiol 1967; 22: 395-401.

14 Woolcock AJ, Vincent NJ, Macklem PT. Frequency dependence of compliance as a test for obstruction in the small airways. J Clin Invest 1969; 48: 1097-1106.

15 Levine G, Housley E, MacLeod P, et al. Gas exchange abnormalities in mild bronchitis and asymptomatic asthma. N Engl J Med 1970; 282: 1277-1282.

16 Despas PJ, Leroux M, Macklem PT. Site of airway obstruction in asthma as determined by measuring maximal expiratory flow breathing air and a helium-oxygen mixture. J Clin Invest 1972; 51: 3235-3243.

17 Cosio M, Ghezzo H, Hogg JC, et al. The relations between structural changes in small airways and pulmonary-function tests. N Engl J Med 1978; 298: 1277-1281.

18 McNulty W, Usmani OS. Techniques of assessing small airways dysfunction. Eur Clin Respir J 2014 ; $1: 25898$.

19 Contoli M, Bousquet J, Fabbri LM, et al. The small airways and distal lung compartment in asthma and COPD: a time for reappraisal. Allergy 2010; 65: 141-151.

20 Collard P, Njinou B, Nejadnik B, et al. Single breath diffusing capacity for carbon monoxide in stable asthma. Chest 1994; 105: 1426-1429.

21 Wenzel SE, Vitari CA, Shende M, et al. Asthmatic granulomatosis: a novel disease with asthmatic and granulomatous features. Am J Respir Crit Care Med 2012; 186: 501-507.

22 Ueda T, Niimi A, Matsumoto $\mathrm{H}$, et al. Role of small airways in asthma: investigation using high-resolution computed tomography. J Allergy Clin Immunol 2006; 118: 1019-1025.

23 Busacker A, Newell JD Jr, Keefe T, et al. A multivariate analysis of risk factors for the air-trapping asthmatic phenotype as measured by quantitative CT analysis. Chest 2009; 135: 48-56.

24 Haldar P, Pavord ID, Shaw DE, et al. Cluster analysis and clinical asthma phenotypes. Am J Respir Crit Care Med 2008; 178: 218-224.

25 Niimi A, Matsumoto H, Amitani R, et al. Effect of short-term treatment with inhaled corticosteroid on airway wall thickening in asthma. Am J Med 2004; 116: 725-731.

26 Walker C, Gupta S, Hartley R, et al. Computed tomography scans in severe asthma: utility and clinical implications. Curr Opin Pulm Med 2012; 18: 42-47.

27 Carroll N, Elliot J, Morton A, et al. The structure of large and small airways in nonfatal and fatal asthma. Am Rev Respir Dis 1993; 147: 405-410.

28 Carroll N, Cooke C, James A. The distribution of eosinophils and lymphocytes in the large and small airways of asthmatics. Eur Respir J 1997; 10: 292-300.

29 Faul JL, Tormey VJ, Leonard C, et al. Lung immunopathology in cases of sudden asthma death. Eur Respir J 1997; 10: 301-307.

30 Suissa S, Ernst P, Benayoun S, et al. Low-dose inhaled corticosteroids and the prevention of death from asthma. N Engl J Med 2000; 343: 332-336.

31 Hamid Q, Song Y, Kotsimbos TC, et al. Inflammation of small airways in asthma. J Allergy Clin Immunol 1997; 100: 44-51.

32 Minshall EM, Hogg JC, Hamid QA. Cytokine mRNA expression in asthma is not restricted to the large airways. J Allergy Clin Immunol 1998; 101: 386-390.

33 Taha RA, Minshall EM, Miotto D, et al. Eotaxin and monocyte chemotactic protein-4 mRNA expression in small airways of asthmatic and nonasthmatic individuals. J Allergy Clin Immunol 1999; 103: 476-483.

34 Balzar S, Wenzel SE, Chu HW. Transbronchial biopsy as a tool to evaluate small airways in asthma. Eur Respir J 2002; 20: 254-259.

35 Kraft M, Djukanovic R, Wilson S, et al. Alveolar tissue inflammation in asthma. Am J Respir Crit Care Med 1996; 154: 1505-1510.

36 Kraft M, Martin RJ, Wilson S, et al. Lymphocyte and eosinophil influx into alveolar tissue in nocturnal asthma. Am J Respir Crit Care Med 1999; 159: 228-234.

37 Andersson CK, Bergqvist A, Mori M, et al. Mast cell-associated alveolar inflammation in patients with atopic uncontrolled asthma. J Allergy Clin Immunol 2011; 127: 905-912.

38 Balzar S, Fajt ML, Comhair SA, et al. Mast cell phenotype, location, and activation in severe asthma. Data from the Severe Asthma Research Program. Am J Respir Crit Care Med 2011; 183: 299-309.

39 Wenzel SE, Szefler SJ, Leung DY, et al. Bronchoscopic evaluation of severe asthma. Persistent inflammation associated with high dose glucocorticoids. Am J Respir Crit Care Med 1997; 156: 737-743.

40 Wenzel SE, Schwartz LB, Langmack EL, et al. Evidence that severe asthma can be divided pathologically into two inflammatory subtypes with distinct physiologic and clinical characteristics. Am J Respir Crit Care Med 1999; 160: 1001-1008.

41 Brown HM. Treatment of chronic asthma with prednisolone; significance of eosinophils in the sputum. Lancet 1958; 2: 1245-1247. 
Pavord ID, Brightling CE, Woltmann G, et al. Non-eosinophilic corticosteroid unresponsive asthma. Lancet 1999; 353: 2213-2214.

Leckie MJ, ten Brinke A, Khan J, et al. Effects of an interleukin-5 blocking monoclonal antibody on eosinophils, airway hyper-responsiveness, and the late asthmatic response. Lancet 2000; 356: 2144-2148.

Flood-Page PT, Menzies-Gow AN, Kay AB, et al. Eosinophil's role remains uncertain as anti-interleukin-5 only partially depletes numbers in asthmatic airway. Am J Respir Crit Care Med 2003; 167: 199-204.

Miranda C, Busacker A, Balzar S, et al. Distinguishing severe asthma phenotypes: role of age at onset and eosinophilic inflammation. J Allergy Clin Immunol 2004; 113: 101-108.

Moore WC, Meyers DA, Wenzel SE, et al. Identification of asthma phenotypes using cluster analysis in the Severe Asthma Research Program. Am J Respir Crit Care Med 2010; 181: 315-323.

Torgerson DG, Ampleford EJ, Chiu GY, et al. Meta-analysis of genome-wide association studies of asthma in ethnically diverse North American populations. Nat Genet 2011; 43: 887-892.

Siroux V, Basagaña X, Boudier A, et al. Identifying adult asthma phenotypes using a clustering approach. Eur Respir J 2011; 38: 310-317.

Wu W, Bleecker E, Moore W, et al. Unsupervised phenotyping of Severe Asthma Research Program participants using expanded lung data. J Allergy Clin Immunol 2014; 133: 1280-1288.

Woodruff PG, Modrek B, Choy DF, et al. T-helper type 2-driven inflammation defines major subphenotypes of asthma. Am J Respir Crit Care Med 2009; 180: 388-395.

Bel EH, Wenzel SE, Thompson PJ, et al. Oral glucocorticoid-sparing effect of mepolizumab in eosinophilic asthma. N Engl J Med 2014; 371: 1189-1197.

De Boever EH, Ashman C, Cahn AP, et al. Efficacy and safety of an anti-IL-13 mAb in patients with severe asthma: a randomized trial. J Allergy Clin Immunol 2014; 133: 989-996.

Moore WC, Fitzpatrick AM, Li X, et al. Clinical heterogeneity in the severe asthma research program. Ann Am Thorac Soc 2013; 10: Suppl, S118-S124.

Bratton DL, Price M, Gavin L, et al. Impact of a multidisciplinary day program on disease and healthcare costs in children and adolescents with severe asthma: a two-year follow-up study. Pediatr Pulmonol 2001; 31: 177-189. Peytremann-Bridevaux I, Arditi C, Gex G, et al. Chronic disease management programmes for adults with asthma. Cochrane Database Syst Rev 2015; 5: CD007988.

Chung KF, Wenzel SE, Brozek JL, et al. International ERS/ATS guidelines on definition, evaluation and treatment of severe asthma. Eur Respir J 2014; 43: 343-373.

Sheshadri A, Hoffman EA, Castro M. Can computed tomography help us identify asthmatic phenotypes? J Allergy Clin Immunol 2014; 133: 739-740.

Gupta S, Hartley R, Khan UT, et al. Quantitative computed tomography-derived clusters: redefining airway remodeling in asthmatic patients. J Allergy Clin Immunol 2014; 133: 729-738.

Montaudon M, Lederlin M, Reich S, et al. Bronchial measurements in patients with asthma: comparison of quantitative thin-section CT findings with those in healthy subjects and correlation with pathologic findings. Radiology 2009; 253: 844-853.

Travis WD, Costabel U, Hansell DM, et al. An official American Thoracic Society/European Respiratory Society statement: update of the international multidisciplinary classification of the idiopathic interstitial pneumonias. Am J Respir Crit Care Med 2013; 188: 733-748.

Pagnoux C, Guilpain P, Guillevin L. Churg-Strauss syndrome. Curr Opin Rheumatol 2007; 19: 25-32.

Trahan S, Hanak V, Ryu JH, et al. Role of surgical lung biopsy in separating chronic hypersensitivity pneumonia from usual interstitial pneumonia/idiopathic pulmonary fibrosis: analysis of 31 biopsies from 15 patients. Chest 2008; 134: 126-132.

Tansey D, Wells AU, Colby TV, et al. Variations in histological patterns of interstitial pneumonia between connective tissue disorders and their relationship to prognosis. Histopathology 2004; 44: 585-596.

Hoffmann RM, Kruis W. Rare extraintestinal manifestations of inflammatory bowel disease. Inflamm Bowel Dis 2004; 10: 140-147.

Frankel SK, Schwarz MI. The pulmonary vasculitides. Am J Respir Crit Care Med 2012; 186: 216-224.

201-212.

Allen TC. Pathology of small airways disease. Arch Pathol Lab Med 2010; 134: 702-718.

Riscili BP, Wood KL. Noninvasive pulmonary Aspergillus infections. Clin Chest Med 2009; 30: 315-335.

Stevens DA, Moss RB, Kurup VP, et al. Allergic bronchopulmonary aspergillosis in cystic fibrosis - state of the art: Cystic Fibrosis Foundation Consensus Conference. Clin Infect Dis 2003; 37: Suppl. 3, S225-S264.

Breekveldt-Postma NS, Erkens JA, Aalbers R, et al. Extent of uncontrolled disease and associated medical costs in severe asthma - a PHARMO study. Curr Med Res Opin 2008; 24: 975-983.

Ivanova JI, Bergman R, Birnbaum HG, et al. Effect of asthma exacerbations on health care costs among asthmatic patients with moderate and severe persistent asthma. J Allergy Clin Immunol 2012; 129: 1229-1235.

Accordini S, Bugiani M, Arossa W, et al. Poor control increases the economic cost of asthma. A multicentre population-based study. Int Arch Allergy Immunol 2006; 141: 189-198.

Bahadori K, Doyle-Waters MM, Marra C, et al. Economic burden of asthma: a systematic review. BMC Pulm Med 2009; 9: 24.

The ENFUMOSA cross-sectional European multicentre study of the clinical phenotype of chronic severe asthma. European Network for Understanding Mechanisms of Severe Asthma. Eur Respir J 2003; 22: 470-477.

Moore WC, Bleecker ER, Curran-Everett D, et al. Characterization of the severe asthma phenotype by the National Heart, Lung, and Blood Institute's Severe Asthma Research Program. J Allergy Clin Immunol 2007; 119: 405-413.

6 Lieberman P, Patterson R, Kunske R. Complications of long-term steroid therapy for asthma. J Allergy Clin Immunol 1972; 49: 329-336.

Schacke H, Docke WD, Asadullah K. Mechanisms involved in the side effects of glucocorticoids. Pharmacol Ther 2002; 96: 23-43. 

analysis of the Asthma Control Characteristics and Prevalence Survey Studies (ACCESS). J Asthma 2010; 47: 257-262.

79 Demoly P, Annunziata K, Gubba E, et al. Repeated cross-sectional survey of patient-reported asthma control in Europe in the past 5 years. Eur Respir Rev 2012; 21: 66-74. Boulet LP, Vervloet D, Magar Y, et al. Adherence: the goal to control asthma. Clin Chest Med 2012; 33: 405-417. Giraud V, Roche N. Misuse of corticosteroid metered-dose inhaler is associated with decreased asthma stability. Eur Respir J 2002; 19: 246-251.

Chanez P, Wenzel SE, Anderson GP, et al. Severe asthma in adults: what are the important questions? J Allergy Clin Immunol 2007; 119: 1337-1348.

Han Q, Luo Q, Xie JX, et al. Diagnostic yield and postoperative mortality associated with surgical lung biopsy for evaluation of interstitial lung diseases: a systematic review and meta-analysis. J Thorac Cardiovasc Surg 2015; 149 : 1394-1401.

Kreider ME, Hansen-Flaschen J, Ahmad NN, et al. Complications of video-assisted thoracoscopic lung biopsy in patients with interstitial lung disease. Ann Thorac Surg 2007; 83: 1140-1144.

Good JT Jr., Kolakowski CA, Groshong SD, et al. Refractory asthma: importance of bronchoscopy to identify phenotypes and direct therapy. Chest 2012; 141: 599-606.

Groeben H, Grosswendt T, Silvanus M, et al. Lidocaine inhalation for local anaesthesia and attenuation of bronchial hyper-reactivity with least airway irritation. Effect of three different dose regimens. Eur J Anaesthesiol 2000; 17: 672-679.

Wahidi MM, Jain P, Jantz M, et al. American College of Chest Physicians consensus statement on the use of topical anesthesia, analgesia, and sedation during flexible bronchoscopy in adult patients. Chest 2011; 140: 1342-1350.

Workshop summary and guidelines: investigative use of bronchoscopy, lavage, and bronchial biopsies in asthma and other airway diseases. J Allergy Clin Immunol 1991; 88: 808-814.

Humbert M, Robinson DS, Assoufi B, et al. Safety of fibreoptic bronchoscopy in asthmatic and control subjects and effect on asthma control over two weeks. Thorax 1996; 51: 664-669.

Moore WC, Evans MD, Bleecker ER, et al. Safety of investigative bronchoscopy in the Severe Asthma Research Program. J Allergy Clin Immunol 2011; 128: 328-336.

Payne D, McKenzie SA, Stacey S, et al. Safety and ethics of bronchoscopy and endobronchial biopsy in difficult asthma. Arch Dis Child 2001; 84: 423-426.

Ravini M, Ferraro G, Barbieri B, et al. Changing strategies of lung biopsies in diffuse lung diseases: the impact of video-assisted thoracoscopy. Eur Respir J 1998; 11: 99-103.

Raghu G, Collard HR, Egan JJ, et al. An official ATS/ERS/JRS/ALAT statement: idiopathic pulmonary fibrosis: evidence-based guidelines for diagnosis and management. Am J Respir Crit Care Med 2011; 183: 788-824.

Nguyen W, Meyer KC. Surgical lung biopsy for the diagnosis of interstitial lung disease: a review of the literature and recommendations for optimizing safety and efficacy. Sarcoidosis Vasc Diffuse Lung Dis 2013; 30: 3-16.

Woods AQ, Lynch DA. Asthma: an imaging update. Radiol Clin North Am 2009; 47: 317-329.

Samejima J, Tajiri M, Ogura T, et al. Thoracoscopic lung biopsy in 285 patients with diffuse pulmonary disease. Asian Cardiovasc Thorac Ann 2015; 23: 191-197.

Seeger W, Adir Y, Barbera JA, et al. Pulmonary hypertension in chronic lung diseases. J Am Coll Cardiol 2013; 62: Suppl., D109-D116.

Huber H, Koessler K. The pathology of bronchial asthma. Arch Intern Med 1922; 30: 689-760.

Saetta M, Turato G. Airway pathology in asthma. Eur Respir J 2001; 18: Suppl. 34, 18s-23s.

Trejo Bittar HE, Yousem SA, Wenzel SE. Pathobiology of severe asthma. Annu Rev Pathol 2015; 10: $511-545$.

Jenkins HA, Cool C, Szefler SJ, et al. Histopathology of severe childhood asthma: a case series. Chest 2003; 124: $32-41$.

Ordoñez CL, Khashayar R, Wong HH, et al. Mild and moderate asthma is associated with airway goblet cell hyperplasia and abnormalities in mucin gene expression. Am J Respir Crit Care Med 2001; 163: 517-523.

Gordon IO, Husain AN, Charbeneau J, et al. Endobronchial biopsy: a guide for asthma therapy selection in the era of bronchial thermoplasty. J Asthma 2013; 50: 634-641.

Kanoh S, Tanabe T, Rubin BK. IL-13-induced MUC5AC production and goblet cell differentiation is steroid resistant in human airway cells. Clin Exp Allergy 2011; 41: 1747-1756.

Kuyper LM, Pare PD, Hogg JC, et al. Characterization of airway plugging in fatal asthma. Am J Med 2003; 115 : 6-11.

Aikawa T, Shimura S, Sasaki H, et al. Marked goblet cell hyperplasia with mucus accumulation in the airways of patients who died of severe acute asthma attack. Chest 1992; 101: 916-921.

Ordoñez C, Ferrando R, Hyde DM, et al. Epithelial desquamation in asthma: artifact or pathology? Am J Respir Crit Care Med 2000; 162: 2324-2329.

Jeffery PK, Laitinen A, Venge P. Biopsy markers of airway inflammation and remodelling. Respir Med 2000; 94 : Suppl F, S9-S15.

Jeffery PK. Remodeling in asthma and chronic obstructive lung disease. Am J Respir Crit Care Med 2001; 164: S28-S38.

Chu HW, Halliday JL, Martin RJ, et al. Collagen deposition in large airways may not differentiate severe asthma from milder forms of the disease. Am J Respir Crit Care Med 1998; 158: 1936-1944.

Chu HW, Balzar S, Westcott JY, et al. Expression and activation of 15-lipoxygenase pathway in severe asthma: relationship to eosinophilic phenotype and collagen deposition. Clin Exp Allergy 2002; 32: 1558-1565.

James AL, Elliot JG, Jones RL, et al. Airway smooth muscle hypertrophy and hyperplasia in asthma. Am J Respir Crit Care Med 2012; 185: 1058-1064.

3 Kaminska M, Foley S, Maghni K, et al. Airway remodeling in subjects with severe asthma with or without chronic persistent airflow obstruction. J Allergy Clin Immunol 2009; 124: 45-51.

Bentley JK, Hershenson MB. Airway smooth muscle growth in asthma: proliferation, hypertrophy, and migration. Proc Am Thorac Soc 2008; 5: 89-96.

Haley KJ, Sunday ME, Wiggs BR, et al. Inflammatory cell distribution within and along asthmatic airways. Am J Respir Crit Care Med 1998; 158: 565-572. 
116 Balzar S, Chu HW, Strand M, et al. Relationship of small airway chymase-positive mast cells and lung function in severe asthma. Am J Respir Crit Care Med 2005; 171: 431-439.

117 de Magalhães Simões S, dos Santos MA, da Silva Oliveira M, et al. Inflammatory cell mapping of the respiratory tract in fatal asthma. Clin Exp Allergy 2005; 35: 602-611.

118 Sur S, Crotty TB, Kephart GM, et al. Sudden-onset fatal asthma. A distinct entity with few eosinophils and relatively more neutrophils in the airway submucosa? Am Rev Respir Dis 1993; 148: 713-719.

119 Chanez P, Bourdin A, Vachier I, et al. Effects of inhaled corticosteroids on pathology in asthma and chronic obstructive pulmonary disease. Proc Am Thorac Soc 2004; 1: 184-190.

120 Nguyen LT, Lim S, Oates T, et al. Increase in airway neutrophils after oral but not inhaled corticosteroid therapy in mild asthma. Respir Med 2005; 99: 200-207.

121 Ogata-Suetsugu S, Maeyama T, Takeshita M, et al. A case of diffuse large B-cell lymphoma of the lung demonstrating diffuse ground-glass shadows. Ann Thorac Cardiovasc Surg 2011; 17: 591-594. 